\title{
THE DUST IN THE HYDROGEN-POOR EJECTA OF ABELL 30
}

\author{
J.P. HARRINGTON and K.J. BORKOWSKI \\ Department of Astronomy, University of Maryland \\ W.P. BLAIR \\ Department of Physics and Astronomy, The Johns Hopkins University \\ J. BREGMAN \\ NASA/Ames Research Ctr., Moffett Field, CA
}

\begin{abstract}
High-resolution images in [O III] $\lambda 5007$ of the hydrogen-poor knots of Abell 30 reveal comet-like structures which may be indicative of interaction with the stellar wind. In the near IR, new, higher-resolution, K-band images show an equatorial ring of hot dust that corresponds closely to optical knots 2 and 4 of Jacoby and Ford, while their polar knots 1 and 3 show no comparable IR emission. Both the thermal IR emission and the heavy internal extinction of the central star demands an extremely dusty ejecta. Greenstein showed that the UV extinction curve is fit by amorphous carbon. Our comprehensive dust models consider both the UV extinction and the IR emission from a population of carbon grains. The thermal emission from larger grains produces the far IR emission, while the stochastic heating of very small grains to high temperatures is essential to explain the near IR flux. We are able to reproduce the shape of the near IR spectrum with an $a^{-3.0}$ distribution of grain radii which extends down to a minimum grain radius of $8 \AA$.

We have constructed photoionization models of the dusty, hydrogen-poor gas which include the heat input due to photoelectrons ejected from the dust grains. The grain charge is important as is the enhanced photoelectric yield of small grains. We find that heating by grain photoelectrons dominates photoionization heating. This extra heat source is critical for the optical and UV line emission of the knots.
\end{abstract}

